\title{
Transfer Function Identification using Orthogonal Fourier Transform Modeling Functions
}

\author{
Eugene A. Morelli ${ }^{1}$ \\ NASA Langley Research Center, Hampton, Virginia, 23681
}

\begin{abstract}
A method for transfer function identification, including both model structure determination and parameter estimation, was developed and demonstrated. The approach uses orthogonal modeling functions generated from frequency domain data obtained by Fourier transformation of time series data. The method was applied to simulation data to identify continuous-time transfer function models and unsteady aerodynamic models. Model fit error, estimated model parameters, and the associated uncertainties were used to show the effectiveness of the method for identifying accurate transfer function models from noisy data.
\end{abstract}

$\begin{array}{ll}C_{L} & =\text { nondimensional aerodynamic lift coefficient } \\ E[\cdot] & =\text { expectation operator } \\ \mathcal{F}[\cdot] & =\text { Fourier transform operator } \\ \operatorname{Im}[\cdot] & =\text { imaginary part } \\ j & =\text { imaginary number }=\sqrt{-1} \\ J & =\text { cost function } \\ N & =\text { number of data points } \\ P S E & =\text { predicted squared error } \\ \operatorname{Re}[\cdot] & =\text { real part } \\ s & =\text { Laplace variable } \\ t & =\text { time, } \mathrm{s} \\ T & =\text { maneuver length, } \mathrm{s} \\ \alpha & =\text { angle of attack, rad or deg } \\ \theta & =\text { parameter vector } \\ \Sigma & =\text { covariance matrix } \\ \omega & =\text { frequency, rad/s } \\ \text { superscripts } & \\ T & =\text { transpose } \\ \sim & =\text { estimate } \\ \sim & =\text { transform } \\ (i) & =i^{\text {th }} \text { time derivative } \\ -1 & =\text { matrix inverse } \\ & \end{array}$

\footnotetext{
${ }^{1}$ Research Engineer, Dynamic Systems and Control Branch, MS 308, Associate Fellow
} 


$\begin{array}{ll}\underline{\text { subscripts }} & \\ R & =\text { real } \\ C & =\text { complex } \\ s & =\text { quasi-steady } \\ u & =\text { unsteady }\end{array}$

\section{Introduction}

N the excellent textbook entitled Numerical Methods That (Usually) Work, author Forman Acton writes ${ }^{1}$ :

A favorite form of lunacy among aeronautical engineers produces countless attempts to decide what differential equation governs the motion of some physical object, such as a helicopter rotor. In the archetypical experiment, one measures both the input to the blade and its output - that is, motion. Arguing that the system is a "black box" to which they have the input and the output, they now wish to compute the transfer function, another name for the differential equation. The difficulty here is that many quite different differential equations can give rise to nearly the same outputs from identical inputs. Thus discriminating the "true" equation from its brethren is next to impossible.

In general, a variety of mathematical model structures can be used to fit a particular set of input-output data within a selected error tolerance. For the transfer function model form, this translates to the well-known fact that high-quality model fits to measured data can be obtained using many different orders for the numerator and denominator. The critical difference among such models, not mentioned in the quote above, is that higher-order models do not predict as well for new data, because excess model structure generally fits noise, which is not repeatable. On the other hand, a model with insufficient model structure will not capture important characteristics exhibited in the data, and this also adversely affects prediction accuracy.

In much of the literature on transfer function modeling ${ }^{2-6}$, the model structure (equivalently, the order of the transfer function numerator and denominator) is assumed to be known, so that the modeling problem is reduced to parameter estimation. Extensive work has been done on the so-called "order determination problem" or "variable selection problem" ${ }^{97-8}$, which is equivalent to determining the structure of the transfer function model, mainly for discrete-time models, but also for continuous-time models ${ }^{2,8}$. The quote from Professor Acton's book highlights the need for more work in this area. Transfer function models are important in aerodynamic modeling, aircraft simulation, control system design, actuator modeling, and many other applications, so it is important that models identified from measured data be accurate, with good prediction capability. The model must also be identifiable based on a defined criterion that distinguishes various model forms that are essentially equivalent in terms of model fit to the data.

In this paper, a method is developed for identifying transfer function model structure (numerator and denominator orders) as well as the associated unknown model parameters (transfer function coefficients) and associated uncertainties, based on measured data alone. The method uses orthogonal modeling functions derived from finite Fourier transforms of the measured input-output data. The development here is focused on single-input single-output (SISO) transfer function identification, although the approach could also be applied to multi-input multi-output (MIMO) transfer function matrix identification.

All of the experiment design, data analysis, and modeling tasks included in this work were done using system identification software written in MATLAB ${ }^{\circledR}$, called System IDentification Programs for AirCraft, or SIDPAC ${ }^{9}$. SIDPAC is bundled with Ref. [9], and is therefore publicly available.

The next section describes the method used to identify transfer function model structure and estimate the associated model parameters and uncertainties. This section also includes discussion of a statistical modeling metric used for model structure determination, along with a modulating function approach that can be used to handle general non-zero endpoint conditions, and a discussion of real-time implementation. In the Examples section, the modeling approach is applied to a simulation problem where the true model is known and measurement noise is added to the output. Following that, the modeling technique is applied to identify an aerodynamic transfer function for unsteady aerodynamics. Finally, concluding remarks summarize and discuss the results. 


\section{Method}

The method for both model structure determination and parameter estimation is based on first transforming sampled time-domain data into the frequency domain. This transformation is done using a high-accuracy implementation of the finite Fourier transform at arbitrarily-chosen frequencies ${ }^{9,10}$. Next, the complex-valued frequency domain data is orthogonalized and processed using a statistical modeling metric for efficient and automated model structure determination. Finally, the selected orthogonal model terms are transformed back into physically-relevant model terms, and estimated model parameter values and uncertainties are calculated for the final model.

\section{A. Finite Fourier Transform}

The finite Fourier transform is the analytical tool used to transform measured data from the time domain into the frequency domain. For an arbitrary scalar signal $x(t)$ on the time interval $[0, T]$, the finite Fourier transform is defined by

$$
\mathcal{F}[x(t)] \equiv \tilde{x}(\omega) \equiv \int_{0}^{T} x(t) e^{-j \omega t} d t
$$

which can be approximated by

$$
\tilde{x}(\omega) \approx \Delta t \sum_{i=0}^{N-1} x(i) e^{-j \omega i \Delta t}
$$

where $x(i) \equiv x(i \Delta t)$ and $\Delta t$ is a constant sampling interval. The summation in Eq. (2) is defined as the discrete Fourier transform,

$$
X(\omega) \equiv \sum_{i=0}^{N-1} x(i) e^{-j \omega i \Delta t}
$$

so that the finite Fourier transform approximation in Eq. (2) can be written as

$$
\tilde{x}(\omega) \approx X(\omega) \Delta t
$$

Eq. (4) represents a simple Euler approximation to the finite Fourier transform in Eq. (1). Cubic interpolation can be applied to the sampled data $x(i)$ to improve the numerical accuracy of the transformation, and arbitrary frequencies can be selected for the finite Fourier transform calculation. With these modifications, the finite Fourier transform in Eq. (1) can be computed numerically for arbitrary frequencies, with accuracy on the order of the numerical precision of the computer. The calculations are explained in Refs. [9] and [10], and were used to compute the finite Fourier transform in Eq. (1).

When transforming time-domain data into the frequency domain, bias and drift are always removed from each measured time series prior to applying the Fourier transform. This is to avoid spillage from large low-frequency components which can pollute the frequency-domain data at low frequencies of interest ${ }^{9}$.

When a time series has non-zero endpoints, the Fourier transform of the time derivative of the time series requires endpoint correction terms. Using integration by parts, the finite Fourier transform of the time derivative of $x(t)$ is

$$
\mathcal{F}[\dot{x}(t)]=x(T) e^{-j \omega T}-x(0)+j \omega \tilde{x}(\omega)
$$

Similarly, 


$$
\begin{aligned}
\mathcal{F}[\ddot{x}(t)] & =\dot{x}(T) e^{-j \omega T}-\dot{x}(0)+j \omega \mathcal{F}[\dot{x}(t)] \\
& =\dot{x}(T) e^{-j \omega T}-\dot{x}(0)+j \omega\left[x(T) e^{-j \omega T}-x(0)\right]+(j \omega)^{2} \tilde{x}(\omega)
\end{aligned}
$$

and so on for higher-order derivatives. When the magnitude of the data and time derivatives at the endpoints are small, e.g., for a flight maneuver that starts and ends at the same steady trim condition, then this effect is small, and can be ignored. However, the effect cannot be ignored in general, and will involve correction terms requiring up to $(n-1)^{\text {th }}$ order derivatives at the endpoints for the Fourier transform of $n^{\text {th }}$ order derivatives of the time series.

\section{B. Orthogonal Fourier Transform Modeling Functions}

In past work ${ }^{9,11-13}$, real data vectors were orthogonalized to effectively and accurately determine model structure and associated model parameters for general nonlinear aerodynamic models. For transfer function modeling in the frequency domain, the data are complex. This requires slightly different data handling to achieve the orthogonalization necessary for model structure determination, but otherwise, the same approach used for real data can be applied.

The necessary modification for complex data is very simple, and is related to what is called the Euclidean angle between two complex vectors ${ }^{14}$. For an arbitrary complex vector $\boldsymbol{x}_{C}$ with $N$ generally complex elements, a real isometric vector $\boldsymbol{x}_{R}$ with $2 N$ elements can be assembled by stacking the real and imaginary parts of the complex elements of $\boldsymbol{x}_{C}$ :

$$
\boldsymbol{x}_{R}=\left[\begin{array}{c}
\operatorname{Re}\left[\boldsymbol{x}_{C}\right] \\
\operatorname{Im}\left[\boldsymbol{x}_{C}\right]
\end{array}\right]
$$

Treating the real vector $\boldsymbol{x}_{R}$ as the new data vector, all of the orthogonalization techniques and model structure determination techniques developed previously for real data can be applied without modification. The penalty associated with applying the techniques to complex data is simply a doubling of the length of the data vectors involved. The next two subsections give a brief overview of the orthogonal function modeling technique. Further details can be found in Refs. [9], [11]-[13], which are the original sources for the technique.

\section{Generating Orthogonal Modeling Functions}

Mutually orthogonal vectors $\boldsymbol{p}_{1}, \boldsymbol{p}_{2}, \ldots, \boldsymbol{p}_{n_{o}}$ can be generated from real vectors $\boldsymbol{\xi}_{1}, \boldsymbol{\xi}_{2}, \ldots, \boldsymbol{\xi}_{n_{o}}$ using an orthogonalization procedure analogous to Gram-Schmidt orthogonalization for vectors. The process begins by choosing one of the $\xi_{j}$ as the first orthogonal vector, e.g., $p_{1} \equiv \xi_{1}$. In general, any $\xi_{j}$ can be chosen as the first orthogonal vector, without any change in the procedure. The orthogonal vectors are then generated sequentially as

$$
\boldsymbol{p}_{j}=\boldsymbol{\xi}_{j}-\sum_{k=1}^{j-1} \gamma_{k j} \boldsymbol{p}_{k} \quad j=2,3, \ldots, n_{o}
$$

The $\gamma_{k j}$ for $k=1,2, \ldots, j-1$ are scalars determined by multiplying both sides of Eq. (8) by $\boldsymbol{p}_{k}^{T}$, then invoking the mutual orthogonality of the $\boldsymbol{p}_{k}, k=1,2, \ldots, j$, and solving for $\gamma_{k j}$

$$
\gamma_{k j}=\frac{\boldsymbol{p}_{k}^{T} \boldsymbol{\xi}_{j}}{\boldsymbol{p}_{k}^{T} \boldsymbol{p}_{k}} \quad j=2,3, \ldots, n_{o} \quad k=1,2, \ldots, j-1
$$

It can be seen from Eqs. (8)-(9) that each orthogonal vector can be expressed exactly in terms of a linear expansion of the original vectors. The orthogonal vectors are generated sequentially by orthogonalizing the original vectors with respect to the orthogonal vectors already computed. Each orthogonal vector can be considered an 
orthogonalized version of an original vector. Because the original vectors $\boldsymbol{\xi}_{1}, \boldsymbol{\xi}_{2}, \ldots, \boldsymbol{\xi}_{n_{o}}$ are in general functions of measured data, these vectors can be called original functions, and the corresponding orthogonal vectors $\boldsymbol{p}_{1}, \boldsymbol{p}_{2}, \ldots, \boldsymbol{p}_{n_{o}}$ can be called orthogonal functions.

\section{Conversions Between Orthogonal Functions and Original Functions}

If the $\boldsymbol{p}_{j}$ vectors and the $\boldsymbol{\xi}_{j}$ vectors are arranged as columns of matrices $\boldsymbol{P}$ and $\boldsymbol{X}$, respectively, and the $\gamma_{k j}$ are elements in the $k^{\text {th }}$ row and $j^{\text {th }}$ column of an upper triangular matrix $\boldsymbol{G}$ with ones on the diagonal,

$$
\begin{aligned}
\boldsymbol{P}=\left[\begin{array}{llllll}
\boldsymbol{p}_{1} & \boldsymbol{p}_{2} & \cdots & \boldsymbol{p}_{n_{o}}
\end{array}\right] & \boldsymbol{X}=\left[\begin{array}{llll}
\boldsymbol{\xi}_{1} & \boldsymbol{\xi}_{2} & \cdots & \boldsymbol{\xi}_{n_{o}}
\end{array}\right] \\
\boldsymbol{G} & =\left[\begin{array}{ccccc}
1 & \gamma_{12} & \gamma_{13} & \cdots & \gamma_{1 n_{o}} \\
0 & 1 & \gamma_{23} & \cdots & \gamma_{2 n_{o}} \\
0 & 0 & 1 & \cdots & \gamma_{3 n_{o}} \\
\vdots & \vdots & \vdots & \vdots & \vdots \\
0 & 0 & 0 & \cdots & 1
\end{array}\right]
\end{aligned}
$$

then

$$
\boldsymbol{X}=\boldsymbol{P} \boldsymbol{G}
$$

and

$$
\boldsymbol{P}=\boldsymbol{X} \boldsymbol{G}^{-1}
$$

The columns of $\boldsymbol{G}^{-1}$ contain the coefficients for expansion of each orthogonal function (column of $\boldsymbol{P}$ ) in terms of an exact linear expansion in the original functions (columns of $\boldsymbol{X}$ ). Equation (12) can be used to express each orthogonal function in terms of the original functions. The manner in which the orthogonal functions are generated allows them to be decomposed without ambiguity into an expansion of the original functions, which have physical meaning.

The next section describes orthogonal function modeling in the frequency domain for the transfer function identification problem.

\section{Transfer Function Model Structure Determination and Parameter Estimation in the Frequency Domain}

The transfer function model for a response $y(t)$ to an input $u(t)$ for zero initial conditions can be written as:

$$
\frac{\tilde{y}(s)}{\tilde{u}(s)}=\frac{c_{0}+c_{1} s+c_{2} s^{2}+\ldots+c_{m} s^{m}}{1+d_{1} s+d_{2} s^{2}+\ldots+d_{n} s^{n}}
$$

where the $c_{0}, c_{1}, c_{2}, \ldots, c_{m}$ and $d_{1}, d_{2}, \ldots, d_{n}$ coefficients are model parameters, and the selection of terms for the numerator and denominator (which also determines the integer values of $m$ and $n$ ) defines the model structure. Setting $s=j \omega$ gives the equivalent model in terms of the Fourier transform,

$$
\frac{\tilde{y}(\omega)}{\tilde{u}(\omega)}=\frac{c_{0}+c_{1}(j \omega)+c_{2}(j \omega)^{2}+\ldots+c_{m}(j \omega)^{m}}{1+d_{1}(j \omega)+d_{2}(j \omega)^{2}+\ldots+d_{n}(j \omega)^{n}}
$$

Re-arranging, 


$$
\begin{aligned}
\tilde{y}(\omega)= & c_{0} \tilde{u}(\omega)+c_{1}(j \omega) \tilde{u}(\omega)+c_{2}(j \omega)^{2} \tilde{u}(\omega)+\ldots+c_{m}(j \omega)^{m} \tilde{u}(\omega) \\
& -d_{1}(j \omega) \tilde{y}(\omega)-d_{2}(j \omega)^{2} \tilde{y}(\omega)-\ldots-d_{n}(j \omega)^{n} \tilde{y}(\omega)
\end{aligned}
$$

Allowing for noise $\varepsilon$ on the measured output $z$,

$$
\begin{aligned}
\tilde{z}(\omega)= & \tilde{y}(\omega)+\tilde{\varepsilon} \\
\tilde{z}(\omega)= & c_{0} \tilde{u}(\omega)+c_{1}(j \omega) \tilde{u}(\omega)+c_{2}(j \omega)^{2} \tilde{u}(\omega)+\ldots+c_{m}(j \omega)^{m} \tilde{u}(\omega) \\
& -d_{1}(j \omega) \tilde{z}(\omega)-d_{2}(j \omega)^{2} \tilde{z}(\omega)-\ldots-d_{n}(j \omega)^{n} \tilde{z}(\omega)+\tilde{\varepsilon}^{\prime}
\end{aligned}
$$

For identification purposes, this is an equation-error linear regression in the frequency domain with complexvalued regressors. If the complex regressors for selected frequencies are converted to real vectors using Eq. (7), and orthogonalized as described in the previous section, then the form of the orthogonal function model is

$$
z=a_{1} p_{1}+a_{2} p_{2}+\ldots+a_{n_{s}} p_{n_{s}}+\boldsymbol{\varepsilon}
$$

where $z$ is a $2 M$-dimensional vector for the measured response in the frequency domain,

$$
z=\left[\begin{array}{c}
\operatorname{Re}[\tilde{z}(\omega)] \\
\operatorname{Im}[\tilde{z}(\omega)]
\end{array}\right]
$$

and the quantity $\tilde{z}(\boldsymbol{\omega})$ is the complex vector of Fourier transforms for $M$ selected frequencies in the frequency vector $\boldsymbol{\omega}$. The response $z$ is modeled in terms of a linear combination of $n_{s}$ selected orthogonal functions $\boldsymbol{p}_{j}, j=1,2, \ldots, n_{s}$, where $n_{s} \leq n_{o}, n_{o}=n+m+1$, which allows for the possibility that not all of the model terms in Eq. (16) may be needed in the final model. Each $\boldsymbol{p}_{j}$ is an $2 M$-dimensional vector which depends on input or output data and the analysis frequencies. For example,

$$
\boldsymbol{p}_{1} \equiv \boldsymbol{\xi}_{1}=\left[\begin{array}{c}
\operatorname{Re}[\tilde{\boldsymbol{u}}(\boldsymbol{\omega})] \\
\operatorname{Im}[\tilde{\boldsymbol{u}}(\boldsymbol{\omega})]
\end{array}\right]
$$

The $a_{j}, j=1,2, \ldots, n_{s}$ in Eq. (17) are constant model parameters to be determined, and $\boldsymbol{\varepsilon}$ denotes the modeling error vector.

Now define an $M \times n_{s}$ matrix $\boldsymbol{P}$,

$$
\boldsymbol{P}=\left[\boldsymbol{p}_{1}, \boldsymbol{p}_{2}, \ldots, \boldsymbol{p}_{n_{s}}\right]
$$

and let

$$
\boldsymbol{a}=\left[\begin{array}{llll}
a_{1} & a_{2} & \ldots & a_{n_{s}}
\end{array}\right]^{T} \quad \boldsymbol{\varepsilon}=\left[\begin{array}{c}
\operatorname{Re}\left[\tilde{\boldsymbol{\varepsilon}}^{\prime}\right] \\
\operatorname{Im}\left[\tilde{\boldsymbol{\varepsilon}}^{\prime}\right]
\end{array}\right]
$$

Equation (17) can then be written as a standard least squares regression problem,

$$
z=\boldsymbol{P a}+\boldsymbol{\varepsilon}
$$


The error vector $\boldsymbol{\varepsilon}$ is to be minimized in a least squares sense. The goal is to determine $\boldsymbol{a}$ that minimizes the least squares cost function

$$
J=\frac{1}{2} \boldsymbol{\varepsilon}^{T} \boldsymbol{\varepsilon}=\frac{1}{2}(z-\boldsymbol{P a})^{T}(z-\boldsymbol{P a})
$$

The parameter vector estimate $\hat{\boldsymbol{a}}$ that minimizes this cost function is computed as ${ }^{9}$

$$
\hat{\boldsymbol{a}}=\left[\boldsymbol{P}^{T} \boldsymbol{P}\right]^{-1} \boldsymbol{P}^{T} \boldsymbol{z}
$$

The estimated parameter covariance matrix is ${ }^{9}$

$$
\Sigma_{\hat{\boldsymbol{a}}}=E\left[(\hat{\boldsymbol{a}}-\boldsymbol{a})(\hat{\boldsymbol{a}}-\boldsymbol{a})^{T}\right]=\sigma^{2}\left(\boldsymbol{P}^{T} \boldsymbol{P}\right)^{-1}
$$

where the fit error variance $\sigma^{2}$ can be estimated from the residuals

$$
v=z-P \hat{a}
$$

using

$$
\hat{\sigma}^{2}=\frac{1}{\left(2 M-n_{s}\right)}\left[(z-\boldsymbol{P} \hat{\boldsymbol{a}})^{T}(z-\boldsymbol{P} \hat{\boldsymbol{a}})\right]=\frac{\boldsymbol{v}^{T} \boldsymbol{v}}{\left(2 M-n_{s}\right)}
$$

Parameter standard errors are computed as the square root of the diagonal elements of the $\Sigma_{\hat{\boldsymbol{a}}}$ matrix from Eq. (25), using $\hat{\sigma}^{2}$ from Eq. (27). The identified model output $\hat{\boldsymbol{y}}$ is

$$
\hat{\boldsymbol{y}}=\boldsymbol{P} \hat{\boldsymbol{a}}
$$

When the modeling functions are mutually orthogonal, an appropriate model structure can be determined in a straightforward manner, because the explanatory capability of each orthogonal modeling function is completely distinct from all of the others. This decouples the least squares modeling problem, as will be shown now.

For mutually orthogonal modeling functions,

$$
\boldsymbol{p}_{i}^{T} \boldsymbol{p}_{j}=0 \quad, \quad i \neq j \quad, \quad i, j=1,2, \ldots, n_{o}
$$

so that for $\boldsymbol{P}=\left[\begin{array}{llll}\boldsymbol{p}_{1} & \boldsymbol{p}_{2} & \cdots & \boldsymbol{p}_{n_{o}}\end{array}\right], \boldsymbol{P}^{T} \boldsymbol{P}$ is a diagonal matrix with the inner product of the orthogonal functions on the main diagonal. Using Eqs. (20)-(21) and (29) in Eq. (24), the $j^{\text {th }}$ element of the estimated parameter vector $\hat{\boldsymbol{a}}$ is given by

$$
\hat{a}_{j}=\left(\boldsymbol{p}_{j}^{T} z\right) /\left(\boldsymbol{p}_{j}^{T} \boldsymbol{p}_{j}\right) \quad j=1,2, \ldots, n_{o}
$$

Using Eqs. (20)-(21), (29), and (30) in Eq. (23),

$$
\hat{J}=\frac{1}{2}\left[z^{T} z-\sum_{j=1}^{n_{o}}\left(\boldsymbol{p}_{j}^{T} z\right)^{2} /\left(\boldsymbol{p}_{j}^{T} \boldsymbol{p}_{j}\right)\right]
$$


Equation (31) shows that when the modeling functions are orthogonal, the reduction in the least-squares cost function resulting from including the term $a_{j} \boldsymbol{p}_{j}$ in the model depends only on the response variable data $z$ and the added orthogonal modeling function $\boldsymbol{p}_{j}$. The least-squares modeling problem is therefore decoupled, which means each orthogonal modeling function can be evaluated independently in terms of its ability to reduce the least-squares model fit to the data, regardless of which other orthogonal modeling functions are already selected for the model. When the modeling functions $\boldsymbol{p}_{j}$ are instead a non-orthogonal function set, the least-squares problem is coupled, and iterative analysis is required to find the subset of modeling functions for an adequate model structure.

The orthogonal modeling functions to be included in the model are chosen to minimize predicted squared error, $P S E$, defined by ${ }^{15}$

$$
P S E \equiv \frac{(z-\boldsymbol{P} \hat{\boldsymbol{a}})^{T}(z-\boldsymbol{P} \hat{\boldsymbol{a}})}{2 M}+\sigma_{\max }^{2} \frac{n_{s}}{2 M}
$$

or

$$
P S E=\frac{\hat{J}}{M}+\sigma_{\max }^{2} \frac{n_{s}}{2 M}
$$

where $n_{s}$ is the number of orthogonal functions selected for the model, $n_{s} \leq n_{o}$.

The constant $\sigma_{\max }^{2}$ is the upper-bound estimate of the squared error between future data and the model, i.e., the upper-bound mean squared error for prediction cases. The upper bound is used in the model over-fit penalty term to account for the fact that PSE is calculated during the model structure determination stage, when the model structure is not correct. Using $\sigma_{\max }^{2}$ in the model complexity term also results in the PSE metric conservatively estimating the squared error for prediction cases.

A simple estimate of $\sigma_{\max }^{2}$ that is independent of the model structure can be obtained by considering $\sigma_{\max }^{2}$ to be the residual variance estimate for a constant model equal to the mean of the measured response values,

$$
\sigma_{\max }^{2}=\frac{1}{2 M-1} \sum_{i=1}^{2 M}\left[z_{i}-\bar{z}\right]^{2} \quad \bar{z}=\frac{1}{2 M} \sum_{i=1}^{2 M} z_{i}
$$

The PSE in Eq. (33) depends on the mean squared error, $\hat{J} / M$, and a term proportional to the number of terms in the model, $n_{s}$. The latter term prevents overfitting the data with too many model terms, which is detrimental to model prediction accuracy ${ }^{9,15}$. While the mean squared error $\hat{J} / M$ must decrease with the addition of each orthogonal modeling function to the model (by Eq. (31)), the over-fit penalty term $\sigma_{\max }^{2} n_{s} /(2 M)$ must increase with each added model term ( $n_{s}$ increases). Introducing the orthogonal modeling functions into the model in order of most effective to least effective in reducing the mean squared error (quantified by $(1 / M)\left(\boldsymbol{p}_{j}^{T} z\right)^{2} /\left(\boldsymbol{p}_{j}^{T} \boldsymbol{p}_{j}\right)$ for the $j^{\text {th }}$ orthogonal modeling function) results in the PSE metric always having a single global minimum.

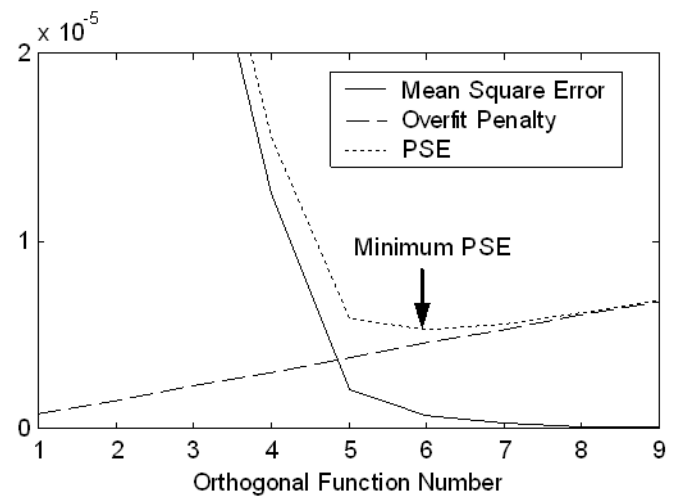

Figure 1. Model structure determination using orthogonal functions and PSE

Figure 1 depicts this graphically, using actual modeling results. The figure shows that after the first 6 modeling functions, the added model complexity associated with the next most effective orthogonal modeling function is not 
justified by the associated reduction in mean squared error. This point is marked by minimum PSE, which defines an adequate model structure with good predictive capability. Ref. [15] contains further statistical arguments and analysis for the form of PSE given in Eq. (33), including justification for its use in modeling problems.

Using orthogonal functions to model the response variable makes it possible to evaluate the merit of including each modeling function individually, using the predicted squared error PSE. The goal is to select a model structure with minimum PSE, and the PSE always has a single global minimum for orthogonal modeling functions ranked according to their effectiveness in reducing the mean squared error. This makes the model structure determination a well-defined and straightforward process that can be (and was) automated. For transfer function model structure determination, selecting appropriate orthogonal functions is equivalent to choosing the appropriate numerator and denominator terms in the model.

After the model structure is determined by selecting orthogonal modeling functions for minimum PSE, the identified model output is

$$
\hat{\boldsymbol{y}}=\boldsymbol{P} \hat{\boldsymbol{a}}
$$

where the $\boldsymbol{P}$ matrix now includes only the $n_{s}$ orthogonal functions selected in the model structure determination, $n_{s} \leq n_{o}$. In general, the retained orthogonal functions will not necessarily be consecutive or the first in the orthogonalization sequence of Eq. (8). Each retained orthogonal function can be decomposed without error into an expansion of the original functions, using the columns of $\boldsymbol{G}^{-1}$ in Eq. (12) corresponding to the retained orthogonal functions. Common terms are combined using double precision arithmetic to arrive finally at a model using only original functions. Terms that contribute less than 0.1 percent of the final model root-mean-square magnitude are dropped.

From Eq. (8), it is clear that the orthogonalization is dependent on the order in which the original functions are processed. If a selected orthogonal function happens to be late in the order, the result is that final conversion from the selected orthogonal function back to original functions will involve small parts of original functions not needed in the final model. To avoid this, orthogonalization is done initially to determine the order of most effective to least effective orthogonal functions, then the associated original functions are re-ordered to match this order of importance, and the orthogonalization is repeated. This simple process results in the minimum number of model terms for the final model in terms of original functions.

The final form of the model is a sum of ordinary functions, with associated model parameter estimates and uncertainty. For the transfer function modeling problem, the model consists of selected terms from the complete set of postulated terms in Eq. (16).

\section{Using Modulating Functions to Avoid Endpoint Corrections}

The finite Fourier transform of time derivatives involves endpoint corrections when the time series endpoints and/or derivatives are non-zero, as shown earlier in Eqs. (5) and (6). These endpoint corrections can be avoided using modulating functions, as first described by Shinbrot ${ }^{16}$. The general idea is to multiply the differential equation associated with Eq. (13) by a suitably-chosen modulating function, then use integration by parts to transfer the derivatives from the data to the modulating function, incurring endpoint terms that involve the modulating functions and its time derivatives evaluated at the endpoints. A smooth differentiable function $\phi(t)$ with the value of the function and its first $n-1$ time derivatives equal to zero at the endpoints is called an $n^{\text {th }}$-order modulating function,

$$
\phi^{(k)}(0)=\phi^{(k)}(T)=0 \quad k=0,1, \ldots, n-1
$$

where $\phi^{(1)}(t)$ denotes the first time derivative of $\phi(t)$, and so on.

Pearson ${ }^{17}$ developed a Fourier-based modulating function. The Fourier-based modulating function for frequency $\omega$ is

$$
\phi(t)=e^{-j \omega t}\left(1-e^{-j \omega_{o} t}\right)^{n} \quad \omega_{o} \equiv 2 \pi / T
$$


The function $\phi(t)$ in Eq. (37) satisfies the requirements of Eq. (36) for an $n^{\text {th }}$-order modulating function. This modulating function can also be written as

$$
\phi(t)=\sum_{k=0}^{n} b_{k} e^{-j\left(\omega+k \omega_{o}\right) t} \quad b_{k}=(-1)^{k} \frac{n !}{k !(n-k) !}
$$

which shows that the modulating function $\phi(t)$ is a summation of complex sinusoidal functions of the same type used in the finite Fourier transform of Eq. (1).

Starting with the differential equation associated with Eq. (16),

$$
z=c_{0} u+c_{1} u^{(1)}+c_{2} u^{(2)}+\ldots+c_{m} u^{(m)}-d_{1} z^{(1)}-d_{2} z^{(2)}-\ldots-d_{n} z^{(n)}+\varepsilon^{\prime}
$$

Multiplying the equation by $\phi(t)$, and integrating over the time period $[0, T]$,

$$
\begin{gathered}
\int_{0}^{T} \phi z d t=c_{0} \int_{0}^{T} \phi u d t+c_{1} \int_{0}^{T} \phi u^{(1)} d t+c_{2} \int_{0}^{T} \phi u^{(2)} d t+\ldots+c_{m} \int_{0}^{T} \phi u^{(m)} d t \\
-d_{1} \int_{0}^{T} \phi z^{(1)} d t-d_{2} \int_{0}^{T} \phi z^{(2)} d t-\ldots-d_{n} \int_{0}^{T} \phi z^{(n)} d t+\tilde{\varepsilon}^{\prime}
\end{gathered}
$$

where $\tilde{\varepsilon}^{\prime}$ represents the complex-valued equation error for the modulated equation. Using integration by parts, and invoking the properties of an $n^{\text {th }}$-order modulating function from Eq. (37),

$$
\begin{aligned}
\int_{0}^{T} \phi z d t= & c_{0} \int_{0}^{T} \phi u d t-c_{1} \int_{0}^{T} \phi^{(1)} u d t+c_{2} \int_{0}^{T} \phi^{(2)} u d t-\ldots+c_{m}(-1)^{m} \int_{0}^{T} \phi^{(m)} u d t \\
& +d_{1} \int_{0}^{T} \phi^{(1)} z d t-d_{2} \int_{0}^{T} \phi^{(2)} z d t+\ldots+d_{n}(-1)^{n+1} \int_{0}^{T} \phi^{(n)} z d t+\tilde{\varepsilon}^{\prime}
\end{aligned}
$$

Each term in Eq. (40) is a complex-valued modulation of the input-output data for frequency $\omega$. Carrying out this process for a vector of frequencies $\omega$ provides frequency-domain data of the same type obtained earlier using the finite Fourier transform. The difference is that using the finite Fourier transform required assuming that the endpoint conditions were close to zero, so that the endpoint terms in Eqs. (5) and (6) would be negligible. Using the modulating function approach, that assumption is not required, because the modulation process removes the non-zero endpoint terms analytically. The modulating function approach therefore allows the use of input-output time series data with arbitrary endpoint conditions.

At this point, the model structure determination and parameter estimation proceed in exactly the same manner as described earlier in Sections II.B. and II.C. Because the modulating function in Eq. (38) uses complex exponential functions, the required modulation integrals in Eq. (40) can be carried out using the same high-accuracy, arbitraryfrequency finite Fourier transform calculation used earlier, ${ }^{9,10}$.

\section{E. Real-Time Implementation}

Transfer function identification in the frequency domain can also be implemented in real time, if the calculations described earlier are applied to frequency-domain data at selected times, typically at regular time intervals. Because the method uses frequency-domain data from the Fourier transform, it is necessary to have values of the Fourier transform available at any time. Fourier transforms can be computed in real time using a recursive Fourier transform, as described next.

For a given frequency $\omega$, the discrete Fourier transform in Eq. (3) at time $i \Delta t$, denoted by $X_{i}(\omega)$, is related to the discrete Fourier transform at time $(i-1) \Delta t$ by 


$$
X_{i}(\omega)=X_{i-1}(\omega)+x(i) e^{-j \omega i \Delta t}
$$

where

$$
e^{-j \omega i \Delta t}=e^{-j \omega \Delta t} e^{-j \omega(i-1) \Delta t}
$$

The quantity $e^{-j \omega \Delta t}$ is constant for a given frequency $\omega$ and constant sampling interval $\Delta t$. It follows that the discrete Fourier transform can be computed for a given frequency at each time step using one addition in Eq. (41) and two multiplications - one in Eq. (42) using the stored constant $e^{-j \omega \Delta t}$ for frequency $\omega$, and one in Eq. (41). There is no need to store the time-domain data in memory when computing the discrete Fourier transform in this way, because the data for each sample time is processed immediately. Time-domain data from the past can be used in all subsequent analysis by simply continuing the recursive calculation of the Fourier transform. In this sense, the recursive Fourier transform implements a memory for spectral information in the data. More data from more maneuvers improves the quality of the data in the frequency domain without increasing memory requirements to store it. Furthermore, the Fourier transform is available at any time $i \Delta t$. The approximation to the finite Fourier transform is completed using Eq. (4).

The recursive computation of the Fourier transform does not use a Fast Fourier Transform $(F F T)$ algorithm ${ }^{18}$, but rather computes the Fourier transform in a straightforward way, using Eqs. (41) and (42). The recursive Fourier transform would therefore be comparatively slow, if the entire frequency band up to the Nyquist frequency $1 /(2 \Delta t)$ were of interest. However, the dynamics for many physical systems (such as rigid-body aircraft dynamics, rotor dynamics, or actuator dynamics) lie in a rather narrow frequency band of approximately 0.01-2.0 Hz. Because the frequency band is limited, it is efficient to compute the discrete Fourier transform using Eqs. (41) and (42) (which represents a recursive formulation of Eq. (3)) for only selected frequencies. With this approach, it is possible to select closely-spaced fixed frequencies for the Fourier transform and the subsequent modeling, and still do the calculations efficiently.

Using a limited frequency band for the Fourier transformation confines the data analysis to the frequency band where the dynamics lie, and automatically filters wide band measurement noise or other dynamics (such as structural responses) outside the frequency band of interest. These automatic filtering features are important for real-time applications, where instrumentation error corrections and noise filtering would require additional computational resources.

In past work using the recursive Fourier transform for stability and control parameter estimation on fighter aircraft and subscale aircraft ${ }^{9,19,20}$, frequency spacing of $0.04 \mathrm{~Hz}$ on an interval of approximately [0.1-2] $\mathrm{Hz}$ was found to be adequate. Finer frequency spacing requires slightly more computation, but was found to have little impact on the results. When the frequency spacing is very coarse, there is a danger of omitting important frequency components, and this can lead to inaccurate parameter estimates. In general, a good rule of thumb is to use frequencies evenly spaced at $0.04 \mathrm{~Hz}$ over the bandwidth for the dynamic system. For good results, the bandwidth should be limited to the frequency range where the signal components in the frequency domain are at least twice the amplitude of the wide band noise level. However, the modeling is robust to these design choices, so the selections to be made are not difficult.

The recursive Fourier transform update need not be done for every sampled time point. Systematically skipping time points effectively lowers the sampling rate of the data prior to Fourier transformation. This saves computation, and does not have a significant adverse impact on the modeling results until the Fourier transform update rate gets below approximately 5 times the highest frequency of interest for the dynamic system. The model structure determination, parameter estimation, and covariance calculations described earlier can be done at any time, but would typically be done at 1 or $2 \mathrm{~Hz}$, to save computations, except in cases of strong nonlinearity, damage, failure, or rapid changes in conditions. For these cases, the model identification update rate can be increased, at the cost of more computations.

For real-time modeling, measured time series are high-pass filtered to remove the steady part of each signal. Then, each perturbation signal is transformed into the frequency domain using the recursive Fourier transform. The break frequency for the high-pass filter is set just below the lowest frequency selected for the modeling and Fourier transform. High-pass filtering can be implemented with a fourth-order Butterworth digital filter, for example. The 
high-pass filtering prevents leakage from the relatively large spectral component at zero frequency, associated with the steady component of each signal, from polluting transformed data at low frequencies.

\section{F. Application to Frequency Responses}

Frequency responses are typically shown using the Bode plot, using separate plots of the magnitude in $\mathrm{dB}$, and the phase angle in degrees, plotted against rotational frequency $\omega$ using a semi-log scale. The frequency response data used to make the Bode plot consists of a vector of complex numbers associated with a vector of frequencies. Although it is simpler and more straightforward to use the finite Fourier transform or Fourier-based modulating functions, it is also possible to use frequency response data with the same modeling approach described in Sections II.B. and II.C. Only a slight modification is required because of the use of cross-spectral density and auto-spectral density in the computation of frequency responses. The analog of Eq. (16) is then

$$
\begin{aligned}
S_{z u}(\omega)= & c_{0} S_{u u}(\omega)+c_{1}(j \omega) S_{u u}(\omega)+c_{2}(j \omega)^{2} S_{u u}(\omega)+\ldots+c_{m}(j \omega)^{m} S_{u u}(\omega) \\
& -d_{1}(j \omega) S_{z u}(\omega)-d_{2}(j \omega)^{2} S_{z u}(\omega)-\ldots-d_{n}(j \omega)^{n} S_{z u}(\omega)+\tilde{\varepsilon}^{\prime}
\end{aligned}
$$

where $\tilde{\varepsilon}^{\prime}$ represents the complex-valued equation error. Using this form, all of the material on transfer function model structure determination and parameter estimation given earlier can be applied without modification.

\section{G. Application to State-Space Model Structure Determination in the Frequency Domain}

The model structure determination approach described in Sections II.B. and II.C. can also be applied to determine model structure for state-space models in the frequency domain. For this application, the procedure is identical to the approach taken in the time domain, described in Ref. [9], except that complex data in the frequency domain is first modified by the simple expression in Eq. [7] as a preliminary step. Beyond that, model structure determination procedures such as stepwise regression and orthogonal function modeling, which depend fundamentally on orthogonalization in function space, can be applied in exactly the same manner as in the time domain. This approach to state-space model structure determination in the frequency domain is more efficient and less subjective than the common practice ${ }^{6}$ of iteratively estimating the parameters in candidate state-space model structures, then retaining or rejecting specific terms based estimated uncertainty measures or parameter insensitivities.

\section{Examples}

The transfer function identification procedure will be demonstrated using simulated data for known transfer functions. Application to identifying unsteady aerodynamic models will also be discussed and demonstrated.

\section{A. Transfer Function Identification from Simulated Data}

An optimized wide-band multisine excitation input ${ }^{9,19-21}$ was applied to a known transfer function:

$$
\frac{\tilde{y}(s)}{\tilde{u}(s)}=\frac{1+0.5 s}{1+0.159 s+0.025 s^{2}}
$$

and the output was corrupted with 5 percent white Gaussian noise. The simulated time series data are shown in the top plot of Figure 2. The transfer function model in Eq. (44) could represent the short-period pitch rate dynamic response to elevator deflections for an airplane, but could also describe many other physical systems.

The transfer function identification procedure was applied to the measured time-domain data, producing the modeling results given in Table 1. The results indicate accurate model structure determination and model parameter estimates, with appropriate and small error bounds for the estimated model parameters. Note that the algorithm identified the correct model structure (transfer function numerator and denominator orders) in addition to accurately estimating the model parameters. Maximum model order equal to 3 was used to generate the candidate orthogonal modeling functions in the frequency domain.

The model fit to the time-domain output data is shown in the middle plot of Figure 2. The lower plot shows the model residuals in the time domain, which are the difference between the measured output and the model output. 
The residuals are essentially white noise, indicating that the modeling process has successfully identified the deterministic functional dependencies in the data.

Figure 3 shows the fit of the identified model to the measured output data in the frequency domain, along with the frequency-domain residual. The plots in Figure 3 use the modulus of the complex-valued frequency-domain quantities, which is the square root of the summed squares of the real and imaginary parts,

$$
|\tilde{y}|=\sqrt{[\operatorname{Re}(\tilde{y})]^{2}+[\operatorname{Im}(\tilde{y})]^{2}}
$$

The input multisine had component frequency content in the frequency band $[0.3,1.8] \mathrm{Hz}$, at evenly-spaced intervals of $0.3 \mathrm{~Hz}$. The analysis frequencies were selected in the frequency band [0.1,2] $\mathrm{Hz}$ at evenly-spaced intervals of $0.1 \mathrm{~Hz}$. There is nothing critical about these selections, except that the analysis frequency band should encompass the frequency content of the multisine input. Many other choices work equally well.

Figures 2 and 3, and the results in Table 1, show that the transfer function identification method produced an accurate transfer function model in both the time domain and the frequency domain, based on the input-output time series data alone. The modulating function approach works equally well, with results from applying that technique also given in Table 1. Model fit to the data using the modulating function approach was very similar to what is shown in Figs. 2 and 3.
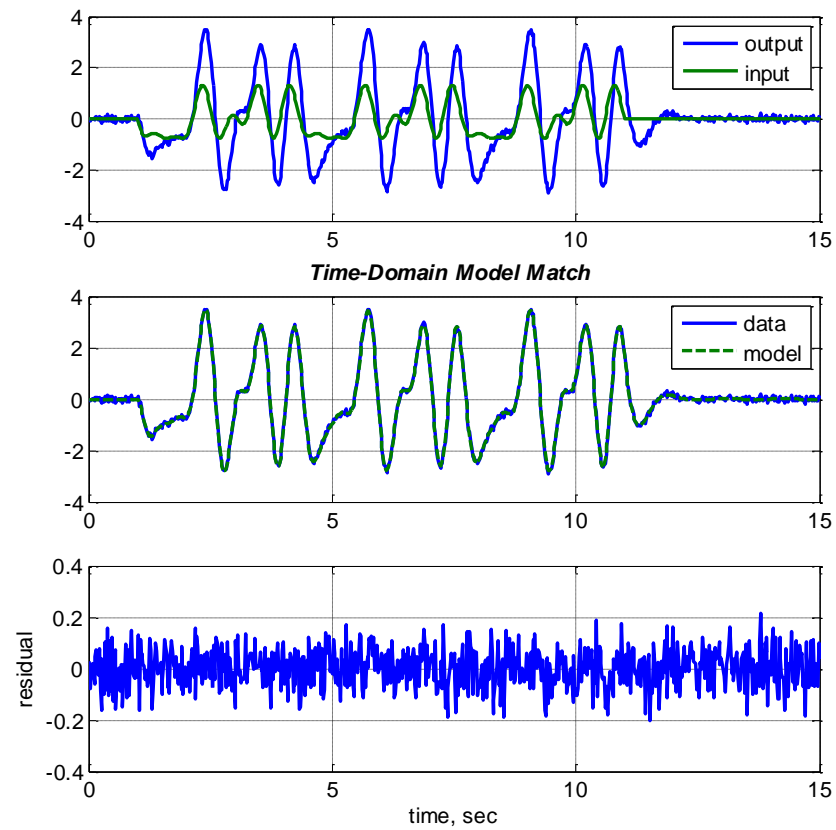

Figure 2. Transfer function identification 

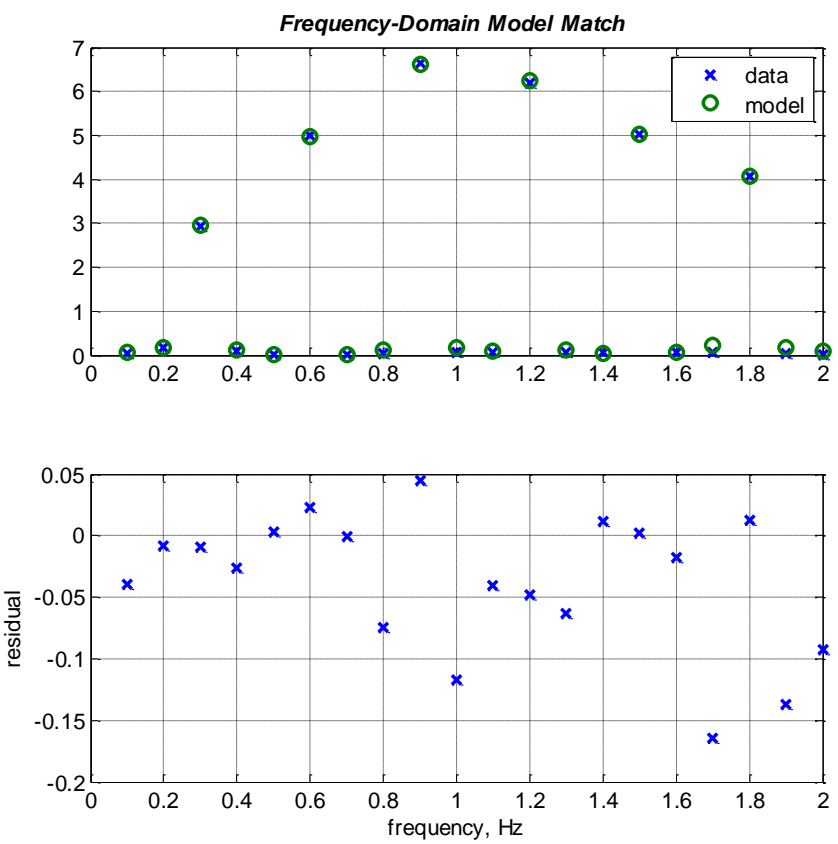

Figure 3. Transfer function model fit in the frequency domain

\section{B. Unsteady Aerodynamic Modeling}

Unsteady aerodynamic models can be identified by applying the equation-error transfer function identification method using orthogonal Fourier transform modeling functions. For this application example, the analysis is confined to the response of lift coefficient $C_{L}$ to angle of attack $\alpha$. The same procedure could be applied to other input-output pairs.

A model for unsteady aerodynamic lift coefficient response to angle of attack can be built based on material in Refs. [22] and [23]. Using a differential equation to implement the unsteady aerodynamics,

$$
\begin{gathered}
C_{L_{u}}=-a \eta \\
\dot{\eta}=-b \eta+\dot{\alpha}
\end{gathered}
$$

where $C_{L_{u}}$ denotes the unsteady part of the lift coefficient and $\eta$ is an additional state associated with the unsteady aerodynamics. Model parameters $a$ and $b$ in general depend on the nominal angle of attack $\alpha_{o}$. The differential equation model form above is equivalent to an indicial function model for unsteady lift,

$$
\begin{gathered}
\eta \equiv \int_{0}^{t} e^{-b(t-\tau)} \dot{\alpha}(\tau) d \tau \\
C_{L_{u}}=-a \eta=-a\left[\int_{0}^{t} e^{-b(t-\tau)} \dot{\alpha}(\tau) d \tau\right]
\end{gathered}
$$

where the indicial function is postulated as the simple exponential function $e^{-b t}$. In transfer function form, the model for unsteady lift can be derived from Eqs. (46) as 


$$
\frac{\tilde{C}_{L_{u}}}{\tilde{\alpha}}=-\frac{a s}{s+b}
$$

The unsteady aerodynamic model in Eqs. (46)-(48) describes an addition to the lift coefficient that depends on past values of $\dot{\alpha}$, but fades out with time. The $b$ parameter quantifies duration of the unsteady effect, and the $a$ parameter quantifies the magnitude of the unsteady effect. The unsteady aerodynamics $C_{L_{u}}$ is added to the quasi-steady lift coefficient dependence on angle of attack, which is normally obtained from static wind tunnel tests or stability and control flight tests.

For relatively small and slow variations in angle of attack, the transfer function for the lift coefficient due to angle of attack can be modeled as a constant $C_{L_{\alpha}}$,

$$
\frac{\tilde{C}_{L_{s}}}{\tilde{\alpha}}=C_{L_{\alpha}}
$$

The model in Eq. (49) implements the quasi-steady assumption that the lift coefficient depends only on the current angle of attack. The transfer function for the lift coefficient arising from arbitrary angle of attack variations is the sum of the quasi-steady and unsteady parts,

$$
\frac{\tilde{C}_{L}}{\tilde{\alpha}}=\frac{\tilde{C}_{L_{s}}}{\tilde{\alpha}}+\frac{\tilde{C}_{L_{u}}}{\tilde{\alpha}}=\frac{\left(C_{L_{\alpha}}-a\right) s+C_{L_{\alpha}} b}{(s+b)}
$$

Converting to the form used for transfer function model identification,

$$
\frac{\tilde{C}_{L}}{\tilde{\alpha}}=\frac{C_{L_{\alpha}}+\left(C_{L_{\alpha}}-a\right) b^{-1} s}{1+b^{-1} s}=\frac{c_{0}+c_{1} s}{1+d_{1} s}
$$

This modeling represents the idea of identifying an aerodynamic transfer function for the influence of angle of attack on lift coefficient (cf. Ref. [24]), as opposed to the simple quasi-steady form shown in Eq. (49) that is typically derived from wind tunnel tests or stability and control flight tests.

The top plot in Fig. 4 shows input-output data for simulated unsteady aerodynamics, using the model structure in Eq. (51). The angle of attack was varied using an optimized wide-band excitation input ${ }^{9,19-21}$ and the simulated output was corrupted by 5 percent Gaussian noise. The lower plots in Fig. 4 and both plots in Fig. 5 show the model fit to the data in the time and frequency domains. The input multisine had component frequency content in the frequency band $[0.04,0.68] \mathrm{Hz}$, at evenly-spaced intervals of $0.04 \mathrm{~Hz}$. The analysis frequencies were selected in the frequency band [0.04, 0.82] Hz at evenly-spaced intervals of $0.02 \mathrm{~Hz}$. Again, there is nothing critical about these selections, except that the analysis frequency band should encompass the frequency content of the multisine input.

Table 2 contains the transfer function identification results. As before, the transfer function identification procedure accurately identified both the model structure and the model parameters. Table 2 also contains the values of $a, b$, and $C_{L_{\alpha}}$ used in the model of Eq. (51).

This modeling approach is a practical implementation of the concept of generalizing stability and control derivatives to aerodynamic transfer functions, as described in theory by Etkin ${ }^{24}$, more than 55 years ago. Figure 6 shows the identified aerodynamic transfer function for lift coefficient response to angle of attack from the last example. The plot indicates that the $\tilde{C}_{L} / \tilde{\alpha}$ is not a constant, but rather depends on the frequency of the angle of attack variations.

The same analysis could be applied for more complicated aerodynamic transfer functions, which allows for the possibility of a more general and complicated indicial function than $e^{-b t}$. The model identification in the frequency domain selects the most appropriate model terms, which is the equivalent of identifying a custom indicial function for the unsteady aerodynamics in the frequency domain. 

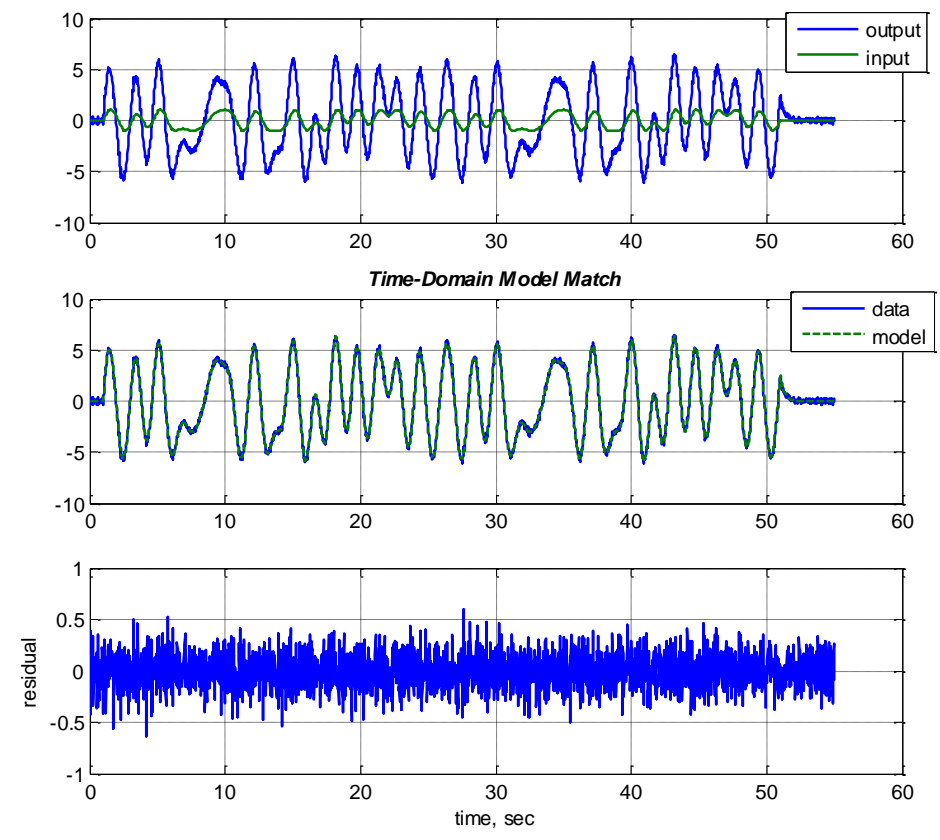

Figure 4. Unsteady aerodynamic transfer function identification
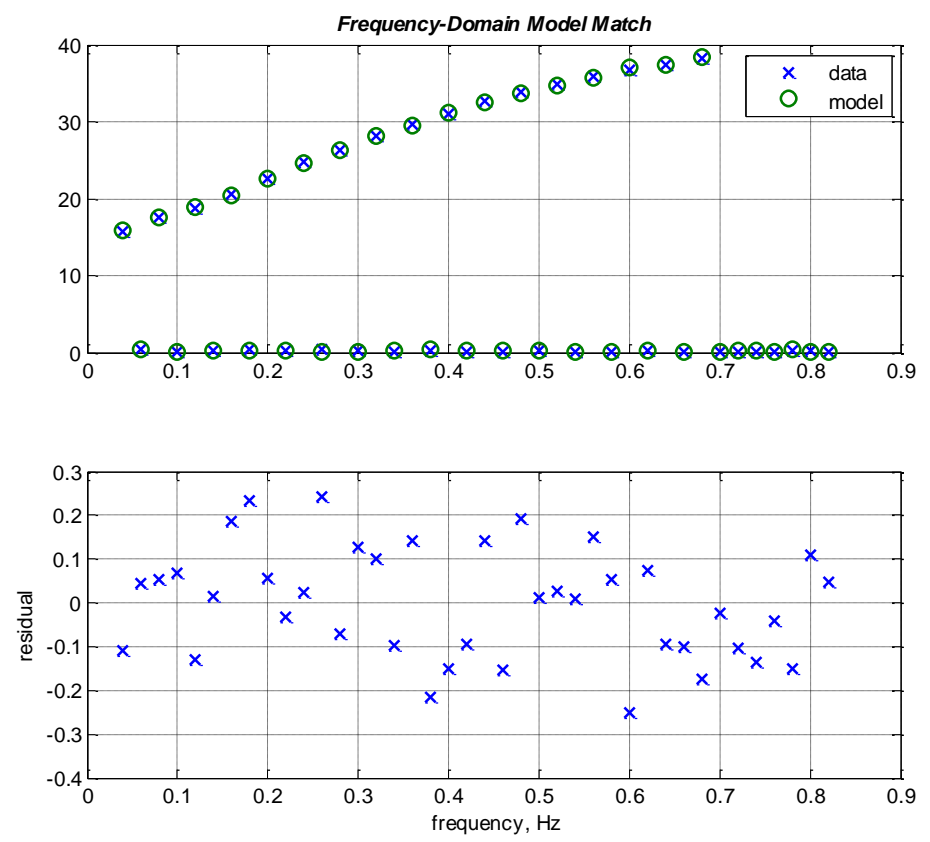

Figure 5. Unsteady aerodynamic transfer function model fit in the frequency domain 

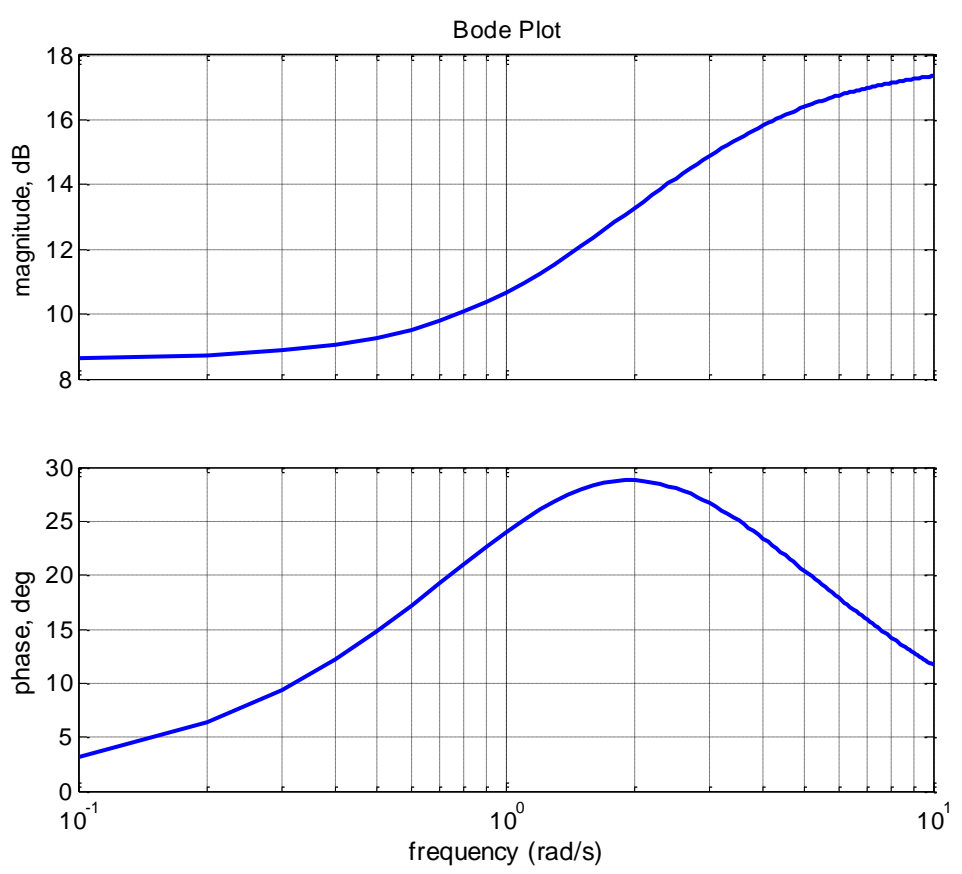

Figure 6. Bode plot of identified $\tilde{C}_{L} / \tilde{\boldsymbol{\alpha}}$ aerodynamic transfer function

\section{Concluding Remarks}

A new method for identifying transfer function models from noisy data was developed and demonstrated using simulated data. The modeling approach uses orthogonal Fourier transform modeling functions in an equation-error formulation in the frequency domain, along with a predicted squared error modeling metric, to identify transfer function model structure and estimate unknown model parameters and uncertainties. Using Fourier-based modulating functions extended the capability of the technique to arbitrary non-zero endpoint conditions. It was also shown that the technique can be applied in real time by using a recursive Fourier transform to transform timedomain data into the frequency domain, then applying the transfer function identification method in the same way as when all the data are analyzed at the same time, in post-flight batch mode. Extensions to frequency response data and application to state-space model identification in the frequency domain were also described. The method was applied to general transfer function identification and unsteady aerodynamic transfer function modeling.

Results from simulation investigations showed that the technique described here can be used effectively for accurate transfer function identification. Model structure and model parameters were identified accurately at the same time, and model parameter uncertainties computed from the data were found to properly characterize the accuracy of the parameter estimates. Further work is required to evaluate the technique on real data.

The approach described here is simple and accurate, and produces high-quality transfer function modeling results from noisy data. Applications include actuator modeling, "black box" transfer function modeling, unsteady aerodynamic modeling, and aerodynamic transfer function modeling.

\section{Acknowledgments}

This research in dynamic modeling is funded by the NASA Aviation Safety Program, Vehicle Systems Safety Technology (VSST) project. 


\section{References}

${ }^{1}$ Acton, F.S. Numerical Methods That (Usually) Work, The Mathematical Association of America, Washington, DC, 1990, pp. 255-256.

${ }^{2}$ Ljung, L. "On the Estimation of Transfer Functions," Automatica, Vol. 21, No. 6, 1985, pp. 677-696.

${ }^{3}$ Pearson, A.E. “Aerodynamic Parameter Estimation Via Fourier Modulating Function Techniques,” NASA CR 4654, April 1995.

${ }^{4}$ Spanos, J.T. and Mingori, D.L. "Newton Algorithm for Fitting Transfer Functions to Frequency Response Measurements," Journal of Guidance, Control, and Dynamics, Vol. 16, No. 1, January-February 1993, pp. 34-39.

${ }^{5}$ Marchand, M. and Fu, K. "Frequency Domain Parameter Estimation of Aeronautical Systems Without and With Time Delay," 7th IFAC Symposium on Identification and System Parameter Estimation, York, UK, 1985.

${ }^{6}$ Tischler, M.B. and Remple, R.K., Aircraft and Rotorcraft System Identification, AIAA Education Series, AIAA, Reston, VA, August 2006.

${ }^{7}$ Guyon, I. and Elisseeff, A. “An Introduction to Variable and Feature Selection," Journal of Machine Learning Research, Vol. 3, 2003, pp. 1157-82.

${ }^{8}$ Wei, H.L., Billings, S.A., and Liu, J. “Term and Variable Selection for Non-Linear System Identification," International Journal of Control, Vol. 77, No. 1, January 2004, pp. 86-110.

${ }^{9}$ Klein, V. and Morelli, E.A., Aircraft System Identification - Theory and Practice, AIAA Education Series, AIAA, Reston, VA, August 2006.

${ }^{10}$ Morelli, E.A. "High Accuracy Evaluation of the Finite Fourier Transform using Sampled Data," NASA TM 110340, June 1997.

${ }^{11}$ Morelli, E.A. "Nonlinear Aerodynamic Modeling using Multivariate Orthogonal Functions," AIAA Paper 93-3636, AIAA Atmospheric Flight Mechanics Conference, Monterey, CA, August 1993.

${ }^{12}$ Morelli, E.A. "Global Nonlinear Parametric Modeling with Application to F-16 Aerodynamics," ACC Paper WP04-2, Paper ID i-98010-2, American Control Conference, Philadelphia, PA, June 1998.

${ }^{13}$ Morelli, E.A. "Efficient Global Aerodynamic Modeling from Flight Data," AIAA-2012-1050, 50 ${ }^{\text {th }}$ AIAA Aerospace Sciences Meeting, Nashville, TN, January 2012.

${ }^{14}$ Scharnhorst, K. “Angles in Complex Vector Spaces,” Acta Applicandae Mathematicae, Vol. 69, 2001, pp. 95-103.

${ }^{15}$ Barron, A.R., "Predicted Squared Error : A Criterion for Automatic Model Selection," Self-Organizing Methods in Modeling, Farlow, S.J., Ed., Marcel Dekker, Inc., New York, NY, 1984, pp. 87-104.

${ }^{16}$ Shinbrot, M. "On the Analysis of Linear and Nonlinear Dynamical Systems from Transient-Response Data," NACA TN 3288, December 1954. .

${ }^{17}$ Pearson, A.E. “Aerodynamic Parameter Estimation Via Fourier Modulating Function Techniques,” NASA CR 4654, April 1995.

${ }^{18}$ Press, W.H., S.A. Teukolsky, W.T. Vettering, and B.R. Flannery Numerical Recipes in FORTRAN: The Art of Scientific Computing, 2nd Ed., Cambridge University Press, New York, NY, 1992, Chapter 10.

${ }^{19}$ Morelli, E.A. "Multiple Input Design for Real-Time Parameter Estimation in the Frequency Domain," Paper REG-360, 13th IFAC Symposium on System Identification, Rotterdam, The Netherlands, August 2003.

${ }^{20}$ Morelli, E.A. and Smith, M.S. "Real-Time Dynamic Modeling - Data Information Requirements and Flight Test Results," Journal of Aircraft, Vol. 46, No. 6, November-December 2009, pp. 1894-1905.

${ }^{21}$ Morelli, E.A. "Flight-Test Experiment Design for Characterizing Stability and Control of Hypersonic Vehicles," Journal of Guidance, Control, and Dynamics, Vol. 32, No. 3, May-June 2009, pp. 949-959.

${ }^{22}$ Murphy, P.C. and Klein, V. "Validation of Methodology for Estimating Aircraft Unsteady Aerodynamic Parameters from Dynamic Wind Tunnel Tests," AIAA-2003-5397, AIAA Atmospheric Flight Mechanics Conference and Exhibit, Austin, TX, August 2003.

${ }^{23}$ Greenwell, D.I. “A Review of Unsteady Aerodynamic Modelling for Flight Dynamics of Manoeuvrable Aircraft,” AIAA2004-5276, AIAA Atmospheric Flight Mechanics Conference and Exhibit, Providence, RI, August 2004.

${ }^{24}$ Etkin, B. H., “Aerodynamic Transfer Functions: An Improvement on Stability Derivatives For Unsteady Flight,' Univ. of Toronto, UTIA Rept. 42, Toronto, ON, Canada, October 1956. 
Table 1. Parameter estimates and uncertainties for a transfer function model identified from simulated data

\begin{tabular}{cccc}
\hline \hline & & $\begin{array}{c}\text { Orthogonal Fourier } \\
\text { Transform Functions }\end{array}$ & $\begin{array}{c}\text { Orthogonal Fourier-Based } \\
\text { Modulating Functions }\end{array}$ \\
\hline Model Parameter & True Value & Estimate \pm Std. Error & Estimate \pm Std. Error \\
\hline$c_{0}$ & 1.000 & $1.003 \pm 0.020$ & $1.007 \pm 0.016$ \\
\hline$c_{1}$ & 0.500 & $0.496 \pm 0.008$ & $0.504 \pm 0.006$ \\
\hline$d_{1}$ & 0.159 & $0.159 \pm 0.002$ & $0.161 \pm 0.002$ \\
\hline$d_{2}$ & 0.0253 & $0.0252 \pm 0.0003$ & $0.0253 \pm 0.0002$ \\
\hline \hline
\end{tabular}

Table 2. Parameter estimates and uncertainties for an unsteady aerodynamic transfer function model identified from simulated data

\begin{tabular}{ccc}
\hline \hline Model Parameter & True Value & Estimate \pm Std. Error \\
\hline$c_{0}$ & 2.700 & $2.699 \pm 0.011$ \\
\hline$c_{1}$ & 2.333 & $2.336 \pm 0.009$ \\
\hline$d_{1}$ & 0.303 & $0.303 \pm 0.002$ \\
\hline$C_{L_{\alpha}}$ & 2.700 & \\
\hline$a$ & -5.000 & \\
\hline$b$ & 3.300 & \\
\hline \hline
\end{tabular}

